\title{
Assessment of Sensitivity and Specificity of Nasopharyngeal and Throat Swabs in Detection of COVID-19 Infection Among Admitted Patients: A Scientific Perspective
}

\author{
Ahmed M. El-Malky ${ }^{1,2,3}$, Ahmed Ali Ahmed', Haya Jamal Albalawi', \\ Abdulmajeed Al Husain, Afnan A. Alshammari', Khalid Saad Alqarni ${ }^{8}$, \\ Marwa Jamal Albalawi ${ }^{9}$
}

${ }^{I}$ Department of Public Health and Community Medicine, Theodor Bilharz Research Institute, Cairo, Egypt

${ }^{2}$ Morbidity and Mortality Review Unit, King Saud University Medical City, Riyadh, Saudi Arabia

Original Article

\author{
${ }^{3}$ Research Chair of Evidence-Based Healthcare and Knowledge Translation, College of \\ Medicine, King Saud University, Riyadh, Saudi Arabia. \\ ${ }^{4}$ Faculty of pharmacy, Alexandria University, Alexandria, Egypt \\ ${ }^{5}$ Undergraduate Student, College of Medicine, Tabuk University, Saudi Arabia \\ ${ }^{6}$ Faculty of Medicine, Prince Sattam Bin Abdulaziz University, Al-kharj, Saudi Arabia \\ ${ }^{7}$ Department of Respiratory Care, College of Applied Medical Sciences in Jubail, Imam \\ Abdulrahman Bin Faisal University- Dammam, Saudi Arabia. \\ ${ }^{8}$ Medical Intern, Faculty of Medicine, University of Tabuk \\ ${ }^{9}$ Lab specialist, King Abdulaziz University, Saudi Arabia, Master of Molecular microbiology, \\ Strathclyde University. UK
}

\begin{abstract}
Background: Early and accurate detection of respiratory viruses (RV) is important for patient management. We have previously shown that self-collected nasal swabs (NS) are feasible and as sensitive as clinician-collected nasal washes for detection of RV, but the additive benefit of self-collected throat swabs is unknown.

Objectives: To test the rise in auto sufficient nasal yields to the throat swabs in patients with upper respiratory (URTI) symptoms for PCR identification of RV.

Study Design: Patients with URTI symptoms self-collected paired polyurethane foam NS and nylon flocked throat swabs and completed a symptom survey. Swabs were tested for 12 RV by real-time reverse transcription (RT)-PCR. Descriptive, McNemar's, and Wilcoxon signed rank statistical tests were used.

Results: The sample was made up of 115 paired swab nasals and throat, with at least 1 specimen being positive for RV (71/115 (62 percent), including 51 positive for both specimens, 17 positive for NS only and 3 favorable for RV only with throat swab. NS was 96 percent sensitive (95 percent CI: 88-99) compared with 76 per cent in throat swabs, $p<0.001$ (95 percent CI: 65-85). The median PCR period threshold (Ct) of 51 concordant samples was lower in NS (25.1) than in swabs of the throat (32.0).

Conclusion: Self-collection of NS was significantly more sensitive than self-collection of throat swabs for detection of RV by RT-PCR. The addition of throat sampling does not appear to increase the diagnostic load in the self-testing setting.
\end{abstract}


Key Words: Assessment sensitivity; nasal; nasopharyngeal; specificity.

Received: 22 November 2020, Accepted: 28 February 2021

Corresponding Author: Ahmed M. Elmalky, PhD, Public Health and Community Medicine Department, Theodor Bilharz Research Institute, Academy of Scientific Research, Ministry of Higher Education, Cairo, Egypt, Tel.: +20 564495895, E-mail: aelmalky@ksu.edu.sa

ISSN: 2090-0740, November 2018, Vol.19, No. 3

\section{INTRODUCTION}

A non-invasive, patient-accepted, and sensitive method for diagnosis of respiratory virus infection (RVI) can have important implications for patient care, epidemiologic studies, and clinical research. Diagnosis of RVI is often limited by the need to get a clinician-collected respiratory sample. However, it may be difficult for ill patients to go to a healthcare facility, and if they do, time to diagnosis may be delayed ${ }^{[1]}$, and they may expose other patients or staff to infection. Identification of sensitive methods for self-collection would address these issues, as well as provide options for community-based assessments of RVI epidemiology and longitudinal monitoring.

Previous experiments have shown the viability, highly-accepted patient sensitivity and/or self-collected nasal swab (NS) selection of the cline $\mathrm{e}^{[2-8]}$. Nasal swab collection is likely. The function of other respiratory specimens obtained by themselves, whether alone or in conjunction with NS, is uncertain. Our previous analysis of contrasting oral gargles self-collected with NS in recipients of symptomatic RVI has indicated lower oral gargles sensitivity ${ }^{[9]}$. While Ip et al. did not equate the two kinds of specimens ${ }^{[10]}$, both self-collected NS and throat swab used in the Population Influenza Report. In our experience there was no measurement of the additional benefit of self-collected throat swabs.

\section{OBJECTIVES}

We hypothesized that throat swabs would not significantly add to the diagnosis of RVI by evaluating self-collected NS and throat swabs in immunocompetent patients with upper respiratory tract infection (URTI) symptoms using reverse-transcription polymerase chain reaction (RT-PCR).

\section{Study design}

\section{Patients}

Immunocompetent patients of the University tertiary hospial with three days or less of URTI symptoms were prospectively enrolled between April 2020 and September 2020. Participants were allowed to participate more than once if their symptoms were $>4$ weeks apart. The KSUMC Institutional Review Board approved the study.

\section{Material and methods}

After informed consent, participants were provided with written instructions and materials for the selfcollection of NS and throat swabs. Participants collected nasal specimens using a polyurethane foam nasal swab (Puritan Medical Products Co., LLC; no. 25-1805-1PFSC2 Arrow) after instillation of $0.5 \mathrm{~mL}$ of normal saline into one nostril and rotating the swab five seconds in the anterior naris as previously described ${ }^{[8]}$. Throat swabs were collected by swabbing the back of the throat and each tonsil area 2-3 times using a nylon flocked swab (Copan Diagnostics, no.502CS01) and placed in universal transport media. The swabs were transferred to the laboratory by study personnel per manufacturer recommendations, and we have previously shown both specimen types to be stable for 7 days at room temperature ${ }^{[8]}$, Participants also filled out a comprehensive symptom survey as previously described ${ }^{[3]}$.

\section{Respiratory virus detection}

Samples were processed in the laboratory as previously described ${ }^{[3]}$, Twelve RV were tested for using the laboratorydeveloped real-time RT-PCR assays: Respiratory syncytial virus, parainfluenza $1-4$, influenza $A$ and $B$, adenovirus, coronavirus, rhinovirus (HRV), metapneumovirus, and bocavirus $^{[11-15]}$.

Samples were considered positive if the PCR cycle threshold $(\mathrm{Ct})$ value was less than 40 based on established cut-offs for laboratory developed tests.

\section{Statistical analysis}

The detection of an RV of each type was deemed to be truly optimistic. For demo diagrams, symptoms, and RV information, informative and summary statistics have been used. For the estimation of importance, the (categorical) rank tests of McNamara and Wilcoxon (paired Ct values) were used.

\section{RESULTS}

There were prospectively one hundred and fifteen NS and neck swabs from 63 (68.2 percent female). The total time of the processing and care of swab was 1 day (IQR $0-1)$. The 21 participants (33.3 percent) sent more than one episode of symptoms of URTI for specimens. A total of 86 (74.8 percent of 115) symptom surveys were conducted.

\section{Symptoms}

At the period of the specimen col- lection the median amount of symptomatic days was two days (IQR 1-3). (Table 1) indicates how many people with different symptoms have been detected and the ratio with each symptom vs. RV. not observed. There was considerable correlation with the presence of rhinorrhea, nasal / sinal inflammation, and sneezing. 


\section{Respiratory virus detection}

Of the 115 paired specimens, 71 (61.7 percent) were positive for either RV in one of them or for both. In both instances, only one RV has been observed. Table 2 illustrates how individual RV is spread and how RV detections and sensitivities by form and specific virus have been broken down. (Table 2) shows. In 68 (59.1\%) of NDs were positive, and in $44(38.3 \%)$ of those pairs $(p<0.001)$ the throat swabs were positive. Although there were low numbers of individual RVs that make statistical research hard, NS demonstrated the same or better sensitivity (adenovirus) as the throat swab (all other RVs). The patients with such signs including sore throat or rhinorrhea did not vary greatly with the sensitivity of the specimen type (data not displayed).

The mean value of CT was 25.9 (IQR 22.5 - 31.3) in the positive samples of the RV relative to 32.5 for the swabs in the throat $(p<0.0001)$ (IQR 26.9-36.2). 17 pairs were accurate just for NS, compared to only 3 pairs valid for throat swabs. In that case, all nasal and throat signs were observed and both of the viruses had elevated $\mathrm{Ct}$ $(33.8,36.2$, and 38.8) values. The median $\mathrm{Ct}$ values were different, depending on the compatibility of the specimens: the median Ct was lower for NS (higher viral concentration) than the other positive specimen for NS, with comparable findings for throat swabs (Figure 1). The median Ct values for NS were different. Median Ct in NS versus gargantuan sponges did not distinguish between evil throat patients and rhinorrhea patients (NS: 26 [IQR 22.8-32.7] and 27 [IQR 23.6-32.2]; neck swab, respectively: 32.8 [IQR 27.8-36] and 33.1 [IQR 27-36.6]). In NS there was no association of $\mathrm{Ct}$ to throat swabs (coefficient of correlation: $0.214, \mathrm{p}=0.13)$.

Table 1: Reported symptoms and association with respiratory virus detection.

\begin{tabular}{|c|c|c|c|c|}
\hline Symptom & Total, N (\%) a & $\mathrm{RV}+, \mathrm{N}(\%) \mathrm{b}$ & RV-, N (\%) b & p-value \\
\hline Respiratory Rhinorrhea & $71(82.6)$ & $51(94.4)$ & $20(62.5)$ & $<0.001 \mathrm{c}$ \\
\hline Nasal/Sinus & 67 (77.9) & $46(85.2)$ & $21(65.6)$ & 0.035 \\
\hline \multicolumn{5}{|l|}{ congestion } \\
\hline Sore Throat & $65(75.6)$ & $42(77.8)$ & $23(71.9)$ & 0.54 \\
\hline Cough & $60(69.8)$ & $41(75.9)$ & $19(59.4)$ & 0.11 \\
\hline Sneezing & $57(66.3)$ & $44(81.5)$ & $13(40.6)$ & $<0.001$ \\
\hline Sputum & $55(64.0)$ & $35(64.8)$ & $20(62.5)$ & 0.83 \\
\hline Any Systemic & $77(89.5)$ & $48(88.9)$ & $29(90.6)$ & $1.0 \mathrm{c}$ \\
\hline Headache & $55(64.0)$ & $38(70.4)$ & $17(53.1)$ & 0.11 \\
\hline Fatigue & $54(62.8)$ & $35(64.8)$ & $19(59.4)$ & 0.61 \\
\hline Myalgia & $38(44.2)$ & $26(48.1)$ & $12(37.5)$ & 0.34 \\
\hline Fever & $30(34.9)$ & $19(35.2)$ & $11(34.4)$ & 0.94 \\
\hline Diarrhea & $8(9.3)$ & $3(5.6)$ & $5(15.6)$ & $0.14 \mathrm{c}$ \\
\hline
\end{tabular}

Table 2: Sensitivity of nasal swab and throat swab specimens by virus type

\begin{tabular}{cccccccc}
\hline \multicolumn{2}{c}{ Respiratory Virus } & \multicolumn{2}{c}{ Number of viruses detected by method } & \multicolumn{2}{c}{ Sensitivity, $\%(95 \% \text { CI })^{*}$} \\
\hline Any & Total, N(\%) & Both positive & NS+, TS - & TS+, NS- & NS & TS & Pvalue \\
HRV & $71(100)$ & 51 & 17 & 3 & $95.8(88.1-99.1)$ & $76.1(64.5-85.4)$ & 0.002 \\
CoV & $39(54.9)$ & 29 & 7 & 3 & 92.3 & 82.1 \\
Flu & $14(19.7)$ & 10 & 4 & 0 & 100 & 71.4 \\
PIV & $7(9.9)$ & 6 & 1 & 0 & 100 & 85.7 \\
MPV & $4(5.6)$ & 2 & 2 & 0 & 100 & 50.0 \\
BoV & $2(2.8)$ & 1 & 1 & 0 & 100 & 50.0 \\
RSV & $2(2.8)$ & 1 & 1 & 0 & 100 & 50.0 \\
ADV & $2(2.8)$ & 1 & 1 & 0 & 100 & 50.0 \\
\hline
\end{tabular}

*P-values and $95 \% \mathrm{CI}$ only calculated for all viruses together given small numbers of other individual respiratory viruses. NS: nasal swab; TS: throat swab; CI: confidence interval; HRV: human rhinovirus; CoV: coronavirus; Flu: influenza A or B; MPV: meta-pneumo-virus; BoV: boca-virus; RSV: respiratory syncytial virus; PIV: parainfluenza virus 1-4; ADV: adenovirus. 


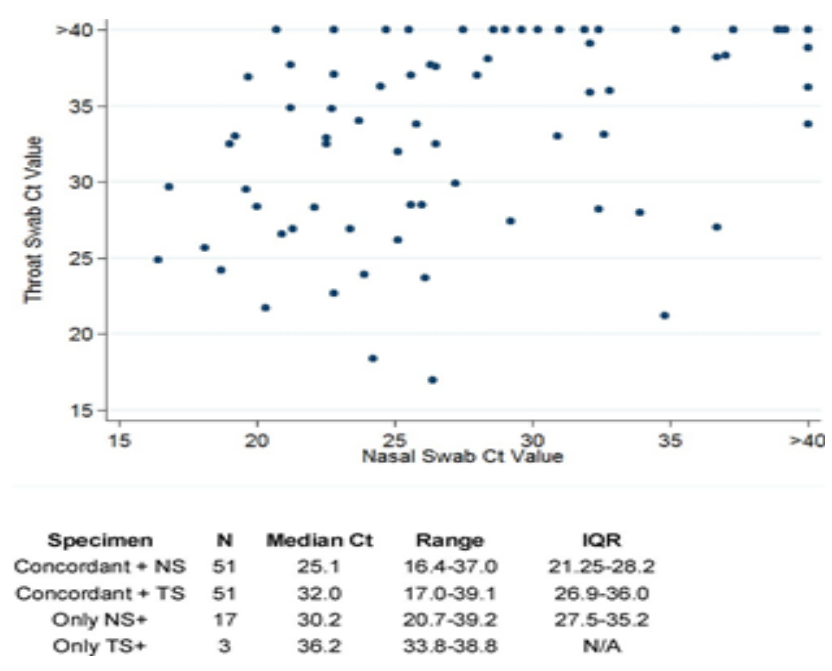

Fig. 1: Comparison of nasal vs. throat swab cycle threshold values in 71 pairs positive by at least one specimen type.

\section{DISCUSSION}

In this prospective study, we have observed that the throat swabs do not greatly lead to the RV identification by RT-PCR in 115 pairs of auto collated polyurethane nasal and nylon flocculated throat sampling in patients with respiratory symptoms. We have noticed $\mathrm{Ct}$ values in NS to be considerably smaller, which suggests higher viral levels. Increased chance that specific symptoms were observed by one approach relative to another (nasal cough, sore throat, etc.), while nasal and sneeze symptoms in those with RV were more frequent compared to those with $\mathrm{RV}$ detected. This research has had limitations. There were several episodes for a variety of viruses, which rendered it impossible to generalize findings for all viruses. In an $\mathrm{H} 5 \mathrm{~N} 1$ analysis, De Jong et al recorded that the pharyngeal specimen collected by the provider is more optimistic and had more viral charges than those collected from nasals ${ }^{[16]}$. We have just 7 un-typed reports of influenza, which implies that this result will not be evaluated. Secondly, while previous data suggest that our self-collected NS equals samples collected by the vendor, we did not test this for throat swabs. This research emphasizes, however, on the real-world application of samples gathered and provides an overview into the virus load and diagnosis in two different breathing areas. Fourth, for nasal and throat (nylon flocked), we used separate swabs. Our previous experiments have demonstrated comparability with providers of collected neuronal washes ${ }^{[8]}$ and at the period the norm of flocked neck swaps; but various styles of swab could deliver different outcomes. Nose swabs are selected based on improved comfort and patience and acceptability.

Overall, in addition to NS, we observed that the collection of throat swabs produced a minimum identification of RVI. Our findings, along with the added responsibility of obtaining a second sample and extra costs for processing, only help the use of self-assembled NS in external and community-based RV testing.

\section{ETHICAL APPROVAL}

Ethical approval was given by the Institutional Review Board of King Saud University Medical City (FH1587 IR\#5149).

\section{AUTHOR CONTRIBUTION}

The design, design, application, analysis and/or writing of the study was a significant commitment by both of their contributors. 1) Formal analyzing, technique, draft, typing, visualization, writing-review. 2) Conceptualization, technique, analysis, editing, tracking. Three, four, five, and six), principles, processes, ratings and edits.

\section{ACKNOWLEDGMENTS}

The scientists are thankful to the research participants. We would like to thank our team for their coordination and participation in this scientific perspective.

\section{CONFLICT OF INTERESTS}

There are no Conflicts of Interest.

\section{REFERENCES}

1. J. Emerson, E. Cochrane, S. McNamara, J. Kuypers, R.L. Gibson, A.P. Campbell, Home self-collection of nasal swabs for diagnosis of acute respiratory virus infec- tions in children with cystic fibrosis, J. Pediatric Infect. Dis. Soc. 2 (4) (2013) 345-351.

2. N. Dhiman, R.M. Miller, J.L. Finley, et al., Effectiveness of patient-collected swabs for influenza testing, Mayo Clin. Proc. 87 (6) (2012) 548-554.

3. C.M. Preiksaitis, J.M. Kuypers, C.E. Fisher, et al., A patient self-collection method for longitudinal monitoring of respiratory virus infection in solid organ transplant recipients, J. Clin. Virol. 62 (2015) 98-102.

4. M.K. Akmatov, A. Gatzemeier, K. Schughart, F. Pessler, Equivalence of self- and staff-collected nasal swabs for the detection of viral respiratory pathogens, PLoS One 7 (11) (2012) e48508.

5. S.B. Lambert, D.M. Whiley, N.T. O’Neill, et al., Comparing nose-throat swabs and nasopharyngeal aspirates collected from children with symptoms for respiratory virus identification using realtime polymerase chain reaction, Pediatrics 122 (3) (2008) e615-620.

6. M.K. Akmatov, S. Krebs, M. Preusse, et al., E-mail-based symptomatic surveillance combined with self-collection of nasal swabs: a new tool for acute respiratory in- fection epidemiology, Int. J. Infect. Dis. 15 (11) (2011) e799-803. 
7. O.E. Larios, B.L. Coleman, S.J. Drews, et al., Selfcollected mid-turbinate swabs for the detection of respiratory viruses in adults with acute respiratory illnesses, PLoS One 6 (6) (2011) e21335.

8. [A.P. Campbell, J. Kuypers, J.A. Englund, K.A. Guthrie, L. Corey, M. Boeckh, Self- collection of foam nasal swabs for respiratory virus detection by PCR among im- munocompetent subjects and hematopoietic cell transplant recipients, J. Clin. Microbiol. 51 (1) (2013) 324-327.

9. C.E. Fisher, R. Bornstein, J. Kuypers, K.R. Jerome, M. Boeckh, A.P. Limaye, Comparison of self-collected nasal swabs with oral washes for sequential viral load monitoring in lung transplant recipients with respiratory virus infection, J. Clin. Virol. 91 (2017) 49-51.

10. D.K. Ip, M. Schutten, V.J. Fang, et al., Validation of self-swab for virologic confirmation of influenza virus infections in a community setting, J. Infect. Dis. 205 (4) (2012) 631-634.

11. J. Kuypers, N. Wright, R. Morrow, Evaluation of quantitative and type-specific real- time RT-PCR assays for detection of respiratory syncytial virus in respiratory spe- cimens from children, J. Clin. Virol. 31 (2) (2004) 123-129.

12. J.Kuypers, N.Wright,L.Corey,R.Morrow,Detection and quantification of human metapneumovirus in pediatric specimens by real-time RT-PCR, J. Clin. Virol. 33 (4) (2005) 299-305.

13. J. Kuypers, N. Wright, J. Ferrenberg, et al., Comparison of real-time PCR assays with fluorescent-antibody assays for diagnosis of respiratory virus infections in children, J. Clin. Microbiol. 44 (7) (2006) 2382-2388.

14. J. Kuypers, E.T. Martin, J. Heugel, N. Wright, R. Morrow, J.A. Englund, Clinical disease in children associated with newly described coronavirus subtypes, Pediatrics. 119 (1) (2007) e70-76.

15. X. Lu, B. Holloway, R.K. Dare, et al., Realtime reverse transcription-PCR assay for comprehensive detection of human rhinoviruses, $\mathrm{J}$. Clin. Microbiol. 46 (2) (2008) 533-539.

16. M.D. de Jong, C.P. Simmons, T.T.Thanh, et al., Fatal outcome of human influenza A(H5N1) is associated with high viral load and hypercytokinemia, Nat. Med. 12 (10) 2006) 1203-1207. 